\title{
The Human Nervous System Structure and Function
}

Sixth Edition 


\section{The Human Nervous System \\ Structure and Function}

Sixth Edition

\section{Charles R. Noback, PhD}

Professor Emeritus

Department of Anatomy and Cell Biology

College of Physicians and Surgeons

Columbia University, New York, NY

\section{Norman L. Strominger, PhD}

Professor

Center for Neuropharmacology and Neuroscience

Department of Surgery (Otolaryngology)

The Albany Medical College

Adjunct Professor, Division of Biomedical Science

University at Albany Institute for Health and the Environment Albany, NY

\section{Robert J. Demarest}

Director Emeritus

Department of Medical Illustration

College of Physicians and Surgeons

Columbia University, New York, NY

\section{David A. Ruggiero, MA, MPhil, PhD}

Professor

Departments of Psychiatry and Anatomy and Cell Biology

Columbia University College of Physicians and Surgeons

New York, NY 
(C) 2005 Humana Press Inc.

999 Riverview Drive, Suite 208

Totowa, New Jersey 07512

www.humanapress.com

All rights reserved. No part of this book may be reproduced, stored in a retrieval system, or transmitted in any form or by any means, electronic, mechanical, photocopying, microfilming, recording, or otherwise without written permission from the Publisher.

All papers, comments, opinions, conclusions, or recommendations are those of the author(s), and do not necessarily reflect the views of the publisher.

This publication is printed on acid-free paper. $\infty$

ANSI Z39.48-1984 (American Standards Institute)

Permanence of Paper for Printed Library Materials.

Production Editor: Tracy Catanese

Cover design by Patricia F. Cleary

Cover Illustration: The cover illustration, by Robert J. Demarest, highlights synapses, synaptic activity, and synaptic-derived proteins, which are critical elements in enabling the nervous system to perform its role.

Neural information derived from sensory receptors, both outside and within the body, activate the spines of the dendrites. As a consequence, neural information is communicated, via the dendrites, to the nucleus of the neuron. This activates the genomic system to release mRNA, which is transported by ribosomes, to the spines. This enables each spine to generate synaptic-specific proteins for the neuron. See Fig. 3.12 for more details

For additional copies, pricing for bulk purchases, and/or information about other Humana titles, contact Humana at the above address or at any of the following numbers: Tel.: 973-256-1699; Fax: 973-256-8341; E-mail: orders@ humanapr.com; or visit our www.humanapress.com

\section{Photocopy Authorization Policy:}

Authorization to photocopy items for internal or personal use, or the internal or personal use of specific clients, is granted by Humana Press, provided that the base fee of US $\$ 30.00$ per copy is paid directly to the Copyright Clearance Center at 222 Rosewood Drive, Danvers, MA 01923. For those organizations that have been granted a photocopy license from the CCC, a separate system of payment has been arranged and is acceptable to Humana Press Inc. The fee code for users of the Transactional Reporting Service is: [1-58829-039-5/05 \$30.00].

eISBN: $1-59259-730-0$

Printed in the United States of America. 10987

Library of Congress Cataloging in Publication Data Library of Congress Cataloging-in-Publication Data

The human nervous system : structure and function / Charles R. Noback ...[et al.].-- 6 th ed. p. $\mathrm{cm}$.

Previous ed. cataloged under: Noback, Charles Robert, 1916-.

Includes bibliographical references and index.

ISBN 1-58829-039-5 (hardcover : alk. paper) -- ISBN 1-58829-040-9 (pbk. : alk. paper)

1. Neurophysiology. I. Noback, Charles Robert, 1916- Human nervous system.

QP361.H85 2005

612.8--dc22 


\section{Dedication}

\section{This book is dedicated to}

Peter, Elizabeth, Norma, and Teddy Noback; Sarah and Evan Bracken; Jessica, William, and Nathan Strominger; Robert and Steven Demarest; Nancy O'Donnell; Anke Lunsmann Nolting and members of the Ruggiero, Mirra, Nolting, von Holsten, Mahler-Welch, and Tanya-Datoek families. 


\section{Preface}

This sixth edition represents the combined efforts of three neuroscientists and a medical illustrator to succinctly present the fundamental principles of the organization, structure, and function of the human nervous system.

The book is intended to meet the basic needs primarily of (1) medical and dental students who want to get a general overview of this discipline; (2) beginning students in the allied health sciences and psychology students who need an introductory yet reasonably comprehensive survey of the subject; (3) residents in neurology, neurosurgery, and neuroradiology who wish to review this subject matter; and (4) readers with a background in biology who want to gain an understanding of some general concepts and specific details.

The text is designed so that the student who is first exposed to neuroscience can get an organized view of the bewilderingly complex human nervous system. The illustrations have been specifically prepared for this book to simplify and clarify items in the text. Clinical correlations and relevant symptoms from lesions are integrated in the text to elucidate important features of the substrate of the brain, spinal cord, and peripheral nervous system. Two chapters specifically deal with "Lesions of the Spinal Nerves" and "Spinal Cord and Lesions of the Brainstem." Each chapter contains a list of selected references as a guide to readers who wish to pursue topics in greater detail. It is anticipated that students who wish further information on specific areas will use the references as a starting point for online searches, which are now readily available. The book incorporates many of the significant recent advances made in neurobiology and molecular biology during the last several years. Information on the dynamics of the dendritic spines, basic neurophysiology, development and growth of the nervous system, auditory and vestibular systems, neurotransmitters as the chemical messengers of certain circuits and pathways, and basal ganglia and extrapyramidal system have been substantially expanded and revised. In addition, some 24 new drawings have been added.

We wish to thank numerous students and colleagues for their many invaluable comments and input. We wish to thank Drs. Krystal Archer-Arroyo, Anthony Cacace, Peter Greene W. Michael King, Lois Laemle; Martha Welch, Sara Glickstein, and Adele, Robert, and Mitchell Strominger, Yuansheng Tan, and Jed Peterson of Albany Medical College, class of 2006, for their efforts on our behalf. We would also like to thank Dr. David Carpenter for his many courtesies.

We thank the people at Humana Press for their patience and good will. It has been a pleasure working with them. We especially want to acknowledge Ms. Donna Niethe for her painstaking efforts in bringing the illustrations into the digital age.

Charles R. Noback Norman L. Strominger David A. Ruggiero Robert J. Demarest 


\title{
Introduction and Terminology
}

\author{
Divisions of the Nervous System \\ Orientation in the Brain \\ Organization of Neurons in the Central Nervous System: \\ Brain and Spinal Cord
}

Most students feel a baffling uncertainty when beginning the study of neuroanatomy. Not until many of the facets of the subject blend in the latter half of the course, do they feel in control over the material. To ameliorate the uncertain feeling, the text and figures in the first five chapters (especially Chap. 1) should be perused for a general understanding only, then used later for reference. Chapters 8 through 13 give basic information about pathway systems, as well as background knowledge for the remaining chapters in the book.

It deserves mention that the nervous system functions together with the endocrine system in harmonizing the many complex activities of the body. The former is a rapid coordinator, whereas the latter is more deliberate in its action.

\section{DIVISIONS OF THE NERVOUS SYSTEM}

The nervous system essentially exhibits a bilateral symmetry with those structural features and pathways located on one side of the midline also found on the other side. It is subdivided anatomically into the central nervous system and the peripheral nervous system and functionally into the somatic nervous system and the autonomic (visceral) nervous system.

The central nervous system (CNS) comprises the brain and spinal cord. The brain is encapsulated within the skull and the spinal cord is at the center of the vertebral column. The peripheral nervous system (PNS) consists of the nerves emerging from the brain (called cranial nerves) and from the spinal cord (called spinal nerves).
The peripheral nerves convey neural messages from (1) the sense organs and sensory receptors in the organism inward to the CNS and from (2) the CNS outward to the muscles and glands of the body.

The somatic nervous system consists of those neural structures of the CNS and PNS responsible for (1) conveying and processing conscious and unconscious sensory (afferent) information, vision, pain, touch, unconscious muscle sense from the head, body wall, and extremities to the CNS and (2) motor (efferent) control of the voluntary (striated) muscles. The autonomic nervous system is composed of the neural structures responsible for (1) conveying and processing sensory input from the visceral organs (e.g., digestive system and cardiovascular system) and (2) motor control of the involuntary (smooth) and cardiac musculature, and of glands of the viscera. Many authors, however, consider the autonomic nervous system to be exclusively concerned with visceral motor activities.

Sensory signals originating in sensory receptors are transmitted through the nervous system along sensory pathways, e.g., pain and temperature pathways and visual pathways. These signals may reach consciousness or may be utilized at unconscious levels. Neural messages for motor activity are conveyed through the nervous system to the muscles and glands along motor pathways. Both the sensory (ascending) and motor (descending) pathways include processing centers (e.g., ganglia, nuclei, laminae, cortices) for each pathway located at different anatomic levels of the spinal cord and brain. The processing centers are the computers of these complex high-speed systems. From receptors in the body 
to the highest centers in the CNS, each sensory pathway system follows, in a general way, a basic sequence: (1) sensory receptors (e.g., touch corpuscles of Meissner in the skin), transmit along (2) nerve fibers to (3) processing centers in the spinal cord and brain, whence signals are conveyed by (4) other nerve fibers, which may ascend on the same side of the CNS or may cross the midline (decussate) and ascend on the opposite side of the CNS before terminating in (5) higher processing centers; from these centers (6) other nerve fibers ascend on the same side before terminating in (7) the highest processing centers in the cerebral cortex. Differences in the basic sequence are present in some ascending systems. In a general way, the motor systems are organized (1) to receive stimuli from the sensory systems at all levels of the spinal cord and brain, and (2) to convey messages via motor pathways to neuromuscular and neuroglandular endings at muscle and gland cells in the head, body, and extremities. The motor pathways comprise (1) sequences of processing centers and their fibers conveying neural influences to other processing centers within the CNS, and (2) the final linkages extending from the CNS via motor nerves of the PNS to muscles and glands.

\section{ORIENTATION IN THE BRAIN}

The long axis through the brain and spinal cord is called the neuraxis. It is shaped in the form of a $T$, with the vertical part being a line passing through the entire spinal cord and brainstem (medulla, pons, and midbrain) and the horizontal part being a line extending from the frontal pole to the occipital pole of the cerebrum (Fig. I.1). In essence, the long axis of the cerebrum is oriented at right angles to the long axis of the brainstem-spinal cord. The term rostral ("toward the beak") means in the direction of the cerebrum. Caudal means in the direction of the coccygeal region. These terms are used in relation to the neuraxis. In this usage, the cerebrum is rostral to the brainstem and the frontal pole of the cerebrum is rostral to the occipital lobe. Horizontal sections through the brainstem are parallel to the neuraxis. Horizontal sections through the cerebrum are cut from the frontal pole to the occipital pole, parallel to a plane passing through both eyes. Horizontal sections through the brainstem and spinal cord are cut rostrocaudally, parallel to the front and back of the neuraxis. A midsagittal or median sagittal section is cut in a vertical plane along the midline; it divides the CNS into symmetric right and left halves. Parasagittal sections are also in the vertical plane, but lateral to the median sagittal section. A coronal section of the cerebrum is cut at a right angle to the horizontal plane. An axial section of the brainstem and spinal cord is cut perpendicular to the neuraxis, giving a cross section or transverse section (see Figs. 7.5 and 13.10). Afferent (or -petal, as in centripetal) refers to bringing to or into a structure such as a nucleus; afferent is often used for sensory. Efferent (or -fugal, as in centrifugal) refers to going away from a structure such as a nucleus; efferent is often used for motor.

\section{ORGANIZATION OF NEURONS IN THE CENTRAL NERVOUS SYSTEM: BRAIN AND SPINAL CORD}

The central nervous system (CNS) comprises gray matter and white matter. Gray matter consists of neuronal cell bodies, dendrites, axon terminals, synapses, and glial cells, and is highly vascular. White matter consists of bundles of axons, many of which are myelinated, and oligodendrocytes; the white color is imparted by the myelin. It lacks neuronal cell bodies and is less vascular than gray matter.

Groupings of neuronal cell bodies within the gray matter are variously known as a nucleus, ganglion, lamina, body, cortex, center, formation, or horn. A cortex is a layer of gray matter on the surface of the brain. Two major cortices are recognized: cerebral and cerebellar cortices. The superior and inferior colliculi of the midbrain and the hippocampal formation also form cortex-like structures. The gray matter, when examined under a microscope, resembles a tangle of nerve and glial processes, called neuropil; this is actually a functionally organized entanglement of processes. Bundles of nerve fibers, many myelinated, are given such special names as tract, fasciculus, brachium, peduncle, lemniscus, commissure, ansa, and capsule. A commissure is a bundle of fibers crossing the midline at right angles to the neuraxis; it usually interconnects similar structures of the two sides of the brain, 


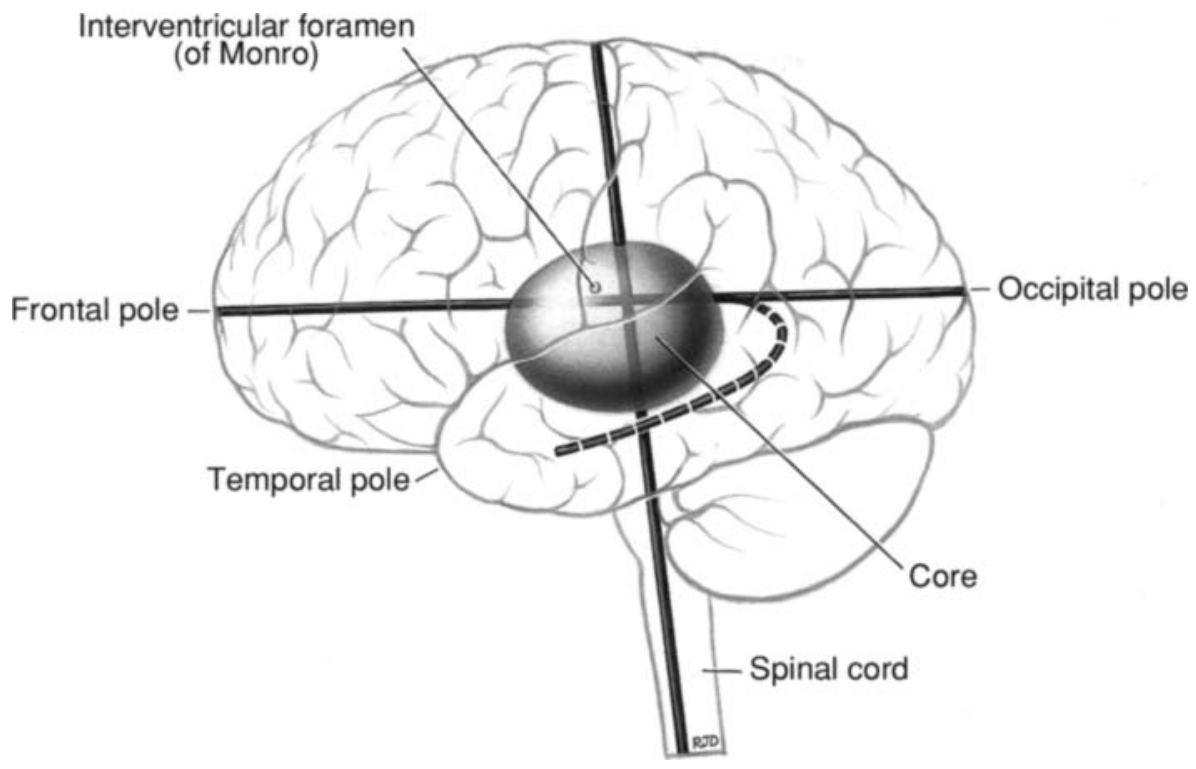

Figure 1: Geometry of the brain. Vertical axis is parallel to the long axis of the brainstem and spinal cord. Horizontal axis is parallel to the cerebrum from frontal pole to occipital pole. The broken arched line illustrates the curving of some structures of the horizontal axis during development exemplified here by the laterally positioned temporal lobe. Core structures along the horizontal axis such as the diencephalon depicted in the drawing are straight. Arching structures that follow a curve include the lateral ventricles, fornix, and hippocampus, among others (see Figs. 1.7 and 5.3).

but sometimes is merely a location where fibers decussate en-route to dissimilar locations.

Contralateral refers to the opposite side; it is used primarily to indicate, for example, that pain or paralysis occurs on the side opposite to that of a lesion. Ipsilateral, or homolateral, refers to the same side; it is used primarily to indicate, for example, that pain or paralysis occurs on the same side as that of a lesion. A modality refers to the quality of a stimulus and the resulting forms of sensation (e.g., touch, pain, sound, vision). Some pathways (tracts, nuclei, or areas of cortex) are somatotopically (topographically) organized; specific portions of these structures are associated with restricted regions of the body. For example, (1) fibers conveying position sense from the hand are in definite locations within the posterior columns (sensory pathway), and (2) certain areas of the motor cortex regulate movements of the thumb. Some structures of the visual pathway are topographically related to specific regions within the retina (retinotopic organization), and similarly some structures of the auditory pathways are organized functionally with respect to different frequencies or tones (tonotopic organization).

\section{Measurements and Dimensions}

The following information is provided for readers who may not have backgrounds in biological or physical sciences. Linear measurements and dimensions generally are given in microns or micrometers $(\mu \mathrm{m})(1 \mu \mathrm{m}$ equals onemillionth of a meter) or nanometers $(\mathrm{nm})(1 \mathrm{~nm}$ or millimicron equals one-billionth of a meter). About $240,000 \mu \mathrm{m}$ equal one inch.

As a reference, the following are the dimensions of some non-neural structures: human ovum, $100 \mu \mathrm{m}$; cross-section of a skeletal muscle fiber, $10-100 \mu \mathrm{m}$; erythrocytes, $8-10 \mu \mathrm{m}$; bacteria, $0.1-8 \mu \mathrm{m}$; and viruses, $0.15-0.5 \mu \mathrm{m}$.

Cell bodies of neurons range from $4-140 \mu \mathrm{m}$ in diameter, nerve fibers (axis cylinders and sheaths) 1 or $2-20 \mu \mathrm{m}$ diameter, chemical synapses $20-30 \mathrm{~nm}$, synaptic vesicles $20-120 \mathrm{~nm}$ and ribosomes are about $15 \mathrm{~nm}$. 


\section{Contents}

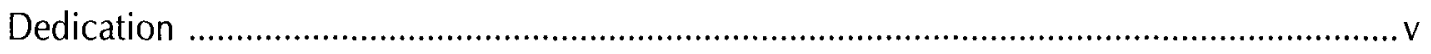

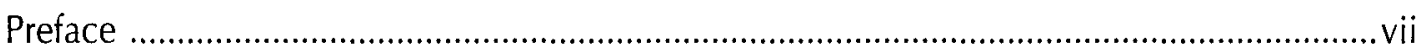

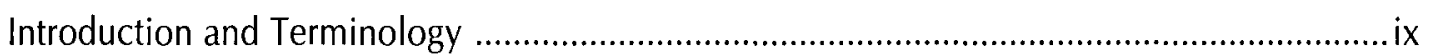

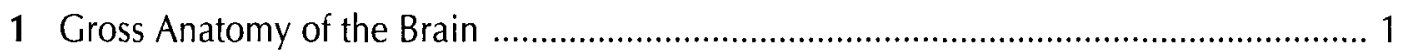

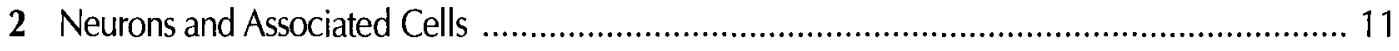

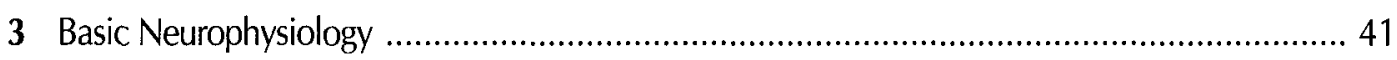

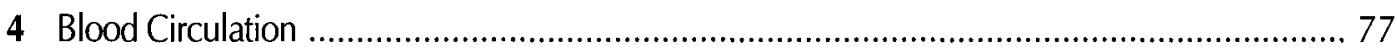

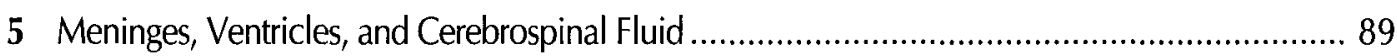

6 Development and Growth of the Nervous System ........................................................ 101

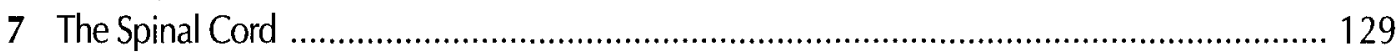

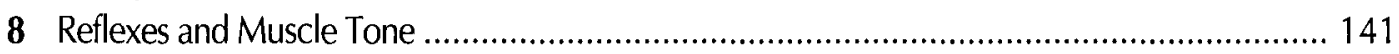

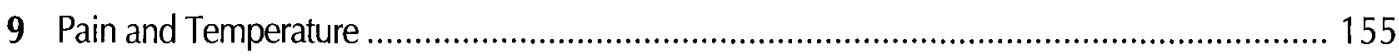

10 Discriminative General Senses, Crude Touch, and Proprioception ...................................... 177

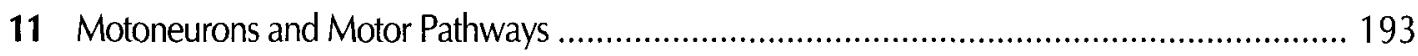

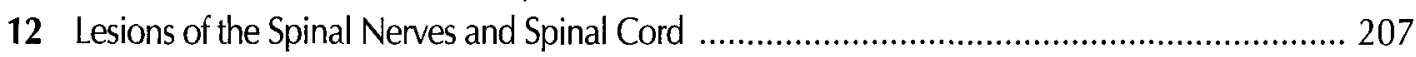

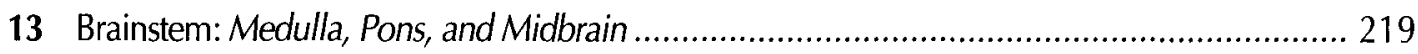

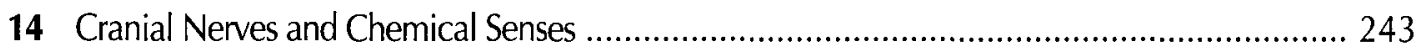

15 Neurotransmitters as the Chemical Messengers of Certain Circuits and Pathways ................... 267

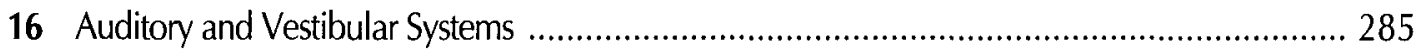

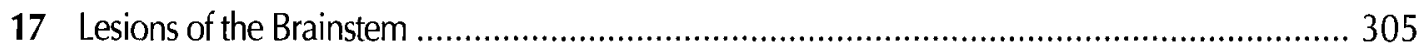

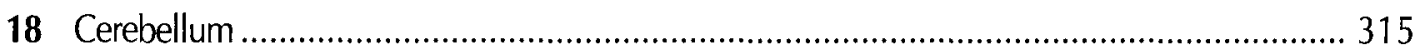

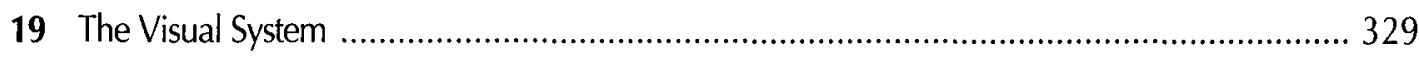

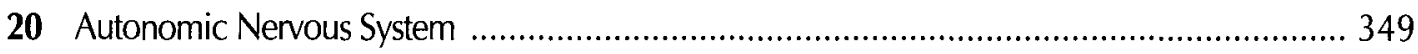

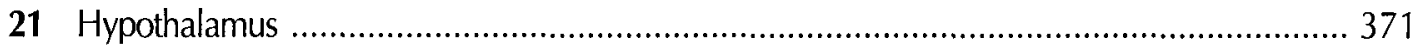

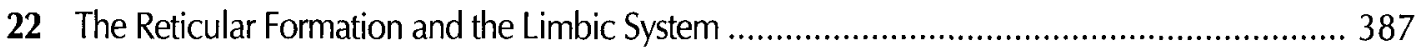

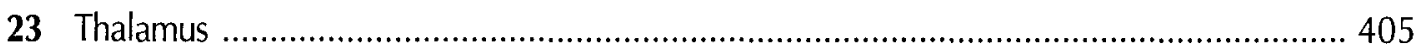

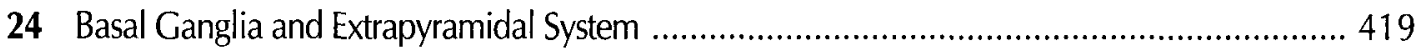

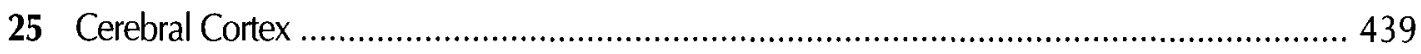

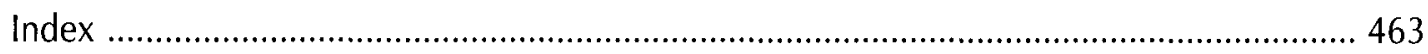

